\title{
eHealth 2015 Special Issue: Impact of Electronic Health Records on the Completeness of Clinical Documen- tation Generated during Diabetic Retinopathy Consultations
}

C. Mitsch'; P. Huber'; K. Kriechbaum¹; C. Scholda'; G. Duftschmid²; T. Wrba²; U. Schmidt-Erfurth ${ }^{1}$

${ }^{1}$ Medical University of Vienna, Department of Ophthalmology and Optometrics, Vienna, Austria; ${ }^{2}$ Medical University of Vienna, Center for Medical Statistics, Informatics, and Intelligent Systems, Vienna, Austria

\section{Keywords}

Ophthalmology, Electronic health records and systems, Testing and evaluation, Encounter notes, Care records

\section{Summary}

Background: Two years ago, the Diabetic Retinopathy (DRP) and Traumatology clinic of the Department of Ophthalmology and Optometrics at the Medical University of Vienna, Austria switched from paper-based to electronic health records. A customized electronic health record system (EHR-S) was implemented.

Objectives: To assess the completeness of information documented electronically compared with manually during patient visits.

Methods: The Preferred Practice Pattern for Diabetic Retinopathy published by the American Academy of Ophthalmology was distilled into a list of medical features grouped into categories to be assessed and documented during the management of patients with DRP. The last seventy paperbased records and all electronic records generated since the switch were analyzed and graded for the presence of features on the list and the resulting scores compared.

Results: In all categories, clinical documentation was more complete in the EHR group. Conclusions: In our setting, the implementation of an EHR-S showed a statistically significant positive impact on documentation completeness.

Correspondence to:

Christoph Mitsch, MD

Department of Ophthalmology and Optometrics

Medical University of Vienna

Waehringer Guertel 18-20

A-1090 Vienna, Austria

Tel.: +43-1-40400-79470

Email: christoph.mitsch@meduniwien.ac.at
Appl Clin Inform 2015; 6: 478-487

http://dx.doi.org/10.4338/ACl-2015-03-RA-0028

received: March 31, 2015

accepted in revised form: May 11, 2015

published: July 29, 2015

Citation: Mitsch C, Huber P, Kriechbaum K, Scholda C, Duftschmid G, Wrba T, Schmidt-Erfurth U. Impact of Electronic Health Records on the Completeness of Clinical Documentation Generated during Diabetic Retinopathy Consultations. Appl Clin Inform $2015 ; 6$ : 478-487 http://dx.doi.org/10.4338/ACl2015-03-RA-0028 


\section{Introduction}

The change from paper to electronic health record systems (EHRs) is driven by the increasing volume of data, the need for it to be easily transferable, and demands for transparency and accountability in the handling of patient information [1]. EHRs not only satisfy these requirements but they have been specified as an essential technology for improving the safety, quality, and efficacy of healthcare [2]. Nevertheless, despite widespread adoption of EHR systems, policies to support exchange of health information and patient engagement still require promotion and ongoing attention [3].

In 2011, Grabenbauer et al. published a qualitative study which identified the factors that influenced the acceptance of two different EHR systems: frequent concerns involved medical workflow and communication [4]. Hoerbst and Ammenwerth systematically reviewed non-functional and functional quality requirements of EHR systems [5]. Aside from high technical quality, sustained completeness of medical documentation before and after the transition to EHRs represents an obvious demand. As an example, Katzer et al. observed an increase in medical documentation after implementing an electronic patient care record in an Emergency Medical Services organization $[6]$.

Various research papers have considered the impact of EHRs on the quality of clinical documentation generated during ophthalmological patient visits. They highlighted significant quantitative and qualitative differences between paper and EHR documentation of ophthalmic findings in the context of the ophthalmological management of age-related macular degeneration (AMD), glaucoma, and pigmented choroidal lesions (PCLs), with EHRs capturing more examination elements than paper records [7]. Pandit et al. reported a positive impact on wait time, an increase in the duration of the clinical examination, and the lack of any change in the patients' perceptions of their visit after an EHR was adopted in a glaucoma subspecialty practice [8]. Another study analyzed the impact of implementing an EHR on ophthalmology operating room management and showed that the system could be adopted without serious negative effects on surgical volume and staffing requirements [9]. However, to our knowledge, no research has been published about the documentation quality using an EHR in a diabetic retinopathy setting.

The outpatient clinic for diabetic retinopathy (DRP) and traumatology at the Medical University of Vienna's Department of Ophthalmology and Optometrics adopted a customized EHR in July 2012. Two years later we compared the quantitative quality of the new system with that of the former paper-based system.

\subsection{Paper-based documentation of diabetic retinopathy consultations at the Department for Ophthalmology and Optometrics}

Clinically relevant details gathered during diabetic retinopathy examinations have traditionally been documented on paper by hand. The resulting medical documentation was then bundled and archived with other documents such as printouts of medical examination devices and administrative forms.

\subsection{The customized electronic health record system implemented for diabetic retinopathy consultations at the Department for Ophthalmol- ogy and Optometrics}

A customized EHR for DRP consultations was developed in collaboration with the Center for Medical Statistics, Informatics, and Intelligent Systems (CEMSIIS). Their software suite "research data and analysis" (RDA) (called ArchiMed in earlier versions) was developed by the CEMSIIS and is currently used in various clinical and research settings at the Medical University of Vienna and its clinical departments at the General Hospital of Vienna (Allgemeines Krankenhaus der Stadt Wien). The system provides a robust framework for building, providing, and analyzing customized forms, documents, and data generated during medical consultations [10]. 
The forms feeding the EHR were developed based on input from ophthalmologists from the diabetic retinopathy department through a series of working group meetings. The resulting forms offer selection lists for most of the categorical variables, such as the presence or severity of pathomorphological findings or diagnoses along with text fields for morphometric and functional attributes. Validation of the form is limited to automated checking of the type of data entered in each field (for example, the system registers an error if a field expects a date and text is entered).

\section{Methods}

This study was performed at the Department of Ophthalmology and Optometrics of the Medical University of Vienna, Austria. The research and measurement methods adhered to the tenets of the Declaration of Helsinki. The study was approved by the local ethics committee and all the patients gave their informed consent to participate in a diabetic retinopathy registry after a detailed discussion with a clinician about the nature and possible consequences of the study procedures.

\subsection{Identification of Cases}

Paper-based clinical documentation was examined by chart review. Administrative hospital databases were inspected to identify individual patient visits for diabetic retinopathy consultations before the implementation of the EHR system. The last 70 paper-based clinical documentations were manually reviewed. All electronic clinical documents produced since the implementation of the EHR system up to the point of our analysis (representing a time span of 24 months) were automatically reviewed and included in our analysis. The discrepancy between the sample sizes stems from a convenience choice due to the increased effort required to examine the paper-based records.

\subsection{Analysis of Cases}

The American Academy of Ophthalmology (AAO) Preferred Practice Pattern (PPP) for the management of diabetic retinopathy [11] was chosen as a reference. A list of required medical features for a thorough ophthalmological exploration of patients with DRP was distilled from the PPP. Furthermore, we categorized the elements to increase their interpretability. See $>$ Table 1 for a list of the medical features and the group categories. Based on this list, we manually analyzed the selected paper-based examination documentations of patient visits created during DRP consultations at the Department of Ophthalmology and Optometrics of the General Hospital of Vienna prior to the implementation of the EHR.

In parallel, all EHRs generated during patient consultations at the department since the implementation of the EHR were analyzed for the presence or absence of each of the elements of the PPPderived list using Microsoft Excel. Elements that represent a systemic property such as a HbAlc result (percent of glycosylated hemoglobin A1 in the patient's blood and a marker for chronically elevated blood sugar levels) could be present once or missing. Thus, these elements could have a maximum score of one point. By contrast, properties of the eyes could be present for one or both eyes or be missing and could therefore have a maximum score of two points.

After the evaluation described above, scores were analyzed using a statistical software package ( $R$ version 3.0.1). For comparison, the 2-tailed unpaired Student's t test was performed which is appropriate for different sample sizes. The Pearson's product-moment correlation coefficient was calculated for the mean scores.

\section{Results}

2,351 EHR documents created between July 2012 and July 2014 and 70 paper notes created during May and June 2012 were examined. The overall mean score for documentation completeness in the paper and the EHR group was 5.43 and 21.25 ( $\mathrm{p}<0.001$, the possible maximum score was 32), respectively. See $>$ Figure1 for a graphical representation of the mean scores.

C) Schattauer 2015 C. Mitsch et al. Impact of EHR on Clinical Documentation during DRP Consultations 
Figure 2 depicts the scores counted in the different categories, and $>$ Table 1 the maximum score, the mean score for both documentation types, and the t-test results. Documentation scores were higher in the EHR than in the paper group in all categories. The differences between paperbased and EHR documentation scores were statistically significant for all the elements documented except visual acuity.

Pearson's product-moment coeffient of correlation of the mean scores was 0.9 . See $>$ Figure 3 for a scatter plot depicting the mean scores of all the documentation elements evaluated: Examination results that were documented more frequently on paper were also documented more often in EHR notes.

\section{Discussion}

We analyzed and graded medical information elements specific for DRP management in paperbased notes and EHR generated documentation during patient visits at the DRP clinic of the Department of Ophthalmology and Optometrics at the Medical University of Vienna, Austria. The completeness of documentation in EHRs was markedly and statistically higher than that of paper notes. Medical documentation generated in this department has been more complete after the switch to the EHR. Additionally, Pearson's product-moment coefficient of the mean scores showed a strong correlation $(\rho=0.9)$ for each of the elements in the paper notes and EHRs. This proves that the relative differences in completeness among the categories were not influenced by introduction of the EHR: The importance of documentation of different medical findings does not depend on the documentation media used, but is influenced rather by medical necessities. For example, in cases where only a longitudinal evaluation of disease features leads to an accurate diagnosis or where diagnosis is still ongoing and symptoms need to be assessed to eliminate possible alternative diagnoses, providers will document more extensively. Medical documentation in this study remains primarily driven and guided by medical judgment: Moreover, in the present hospital information system, the detailed medical services record used for billing purposes is maintained in a separated database.

The implementation of an EHR in a clinical setting signifies much more for the physicians than a transition from pencil-and-paper to keyboard, mouse, and screen. While paper-based documentation is frequently unstructured (mostly similar to the body of Clinical Document Architecture (CDA) Level 1), the degree of granularity of the information structure of most modern EHRs tends to be markedly higher and, at best, allows CDA Level 3 documents to be generated automatically based on the documented data. This data processing functionality, which most healthcare systems seek to implement, apparently has important consequences for the way physicians document data and possibly also for the way in which they work, earlier in the process. This may be because an EHR usually prompts providers by offering a more structured template (covering distinct entities) than blank sheets of paper without guidance on elements that need to be documented or paper forms (providing defined blocks of narrative text). Electronic forms may include check algorithms whereby unfinished or formally flawed form contents cannot be 'saved'. We deliberately precluded this kind of potentially effective control in our EHR, but it's not an inconceivable future upgrade if planned and implemented in close collaboration with all the clinicians who use the system. The EHR used in the DRP clinic allows documentation of elements that are "not assessed" (not measured or examined at the documented visit), "not assessable" (could not be measured or examined, for example details of fundoscopy when a haemorrhage impedes examination of the retina) and the input of an observed biological property. This changes the interpretability of missing information: A paper-based clinical note, which does not contain any comment about a critical feature, for example retinal hemorrhage in DRP, leaves room for ambiguity and thus does not provide robust information about the pathology. The explicit documentation of the absence of a pathological feature strengthens the interpretability of the whole note. It may be time-consuming to document nonpathological properties and to read through a medical patient visit summary containing a high proportion of normal values. A possibility to copy and adapt the values of earlier visits may abridge this process. To speed up comprehension of the resulting greater amount of data, present pathological features can be highlighted in the visual representation of past notes. 
Clinical documentation was more complete in all categories when recorded electronically than on paper. Consequently, the medical information available to the same or another clinician at the next visit will be more comprehensive and continuity of management thus becomes easier and potentially more effective. A more complete documentation of past examinations may mean that the exam does not have to be repeated resulting in economic benefits. Initially some ophthalmologists at the DRP department, who were used to creating notes on paper, resisted the introduction of an EHR but most have since unreservedly acknowledged the system's advantages. Other features such as the ability to immediately print and hand out a well-formatted patient visit summary and the instant transmission of all documents to the hospital information system for convenient access, also contributed to its general acceptance. Furthermore, retrospective analyses based on these data will provide further medical insight into the efficacy and safety of the treatment modalities at the DRP clinic.

\section{Conclusion}

The effect of electronic clinical documentation on completeness of information was positive in our DRP clinic.Our data would support a decision to switch to this modality in departments and offices where the transition has not yet be made.

Clinical Relevance Statement

Our study suggests that the transition from paper-based documentation to EHRs has a positive impact on the completeness of medical documentation.

\section{Conflict of Interest}

The authors declare that they have no conflicts of interest in the research.

\section{Human subjects}

The procedures used have been reviewed in compliance with ethical standards of the responsible committee on human experimentation. 


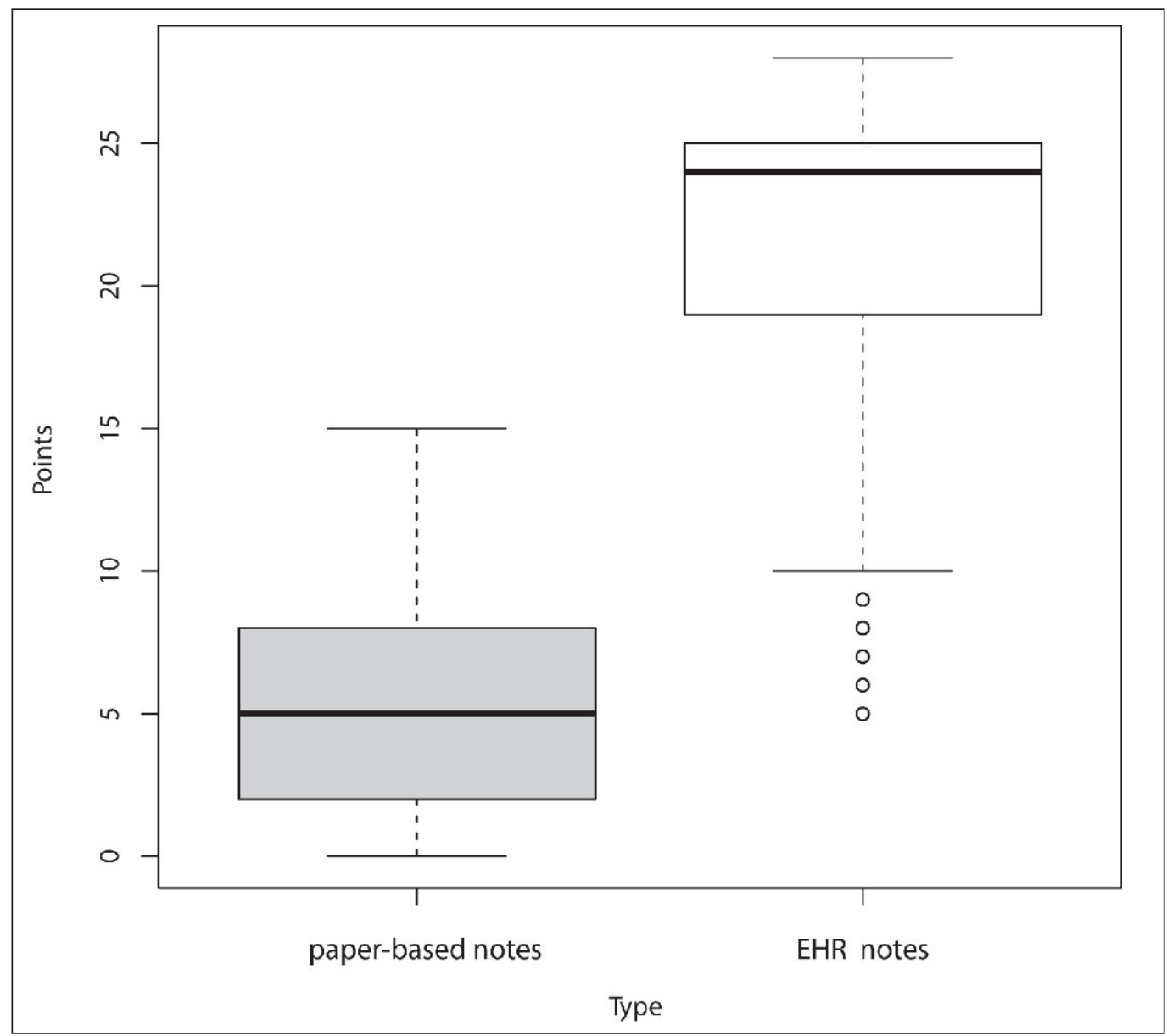

Fig. 1 Box-and-whisker plot of the overall mean documentation score in paper-based and EHR clinical notes 


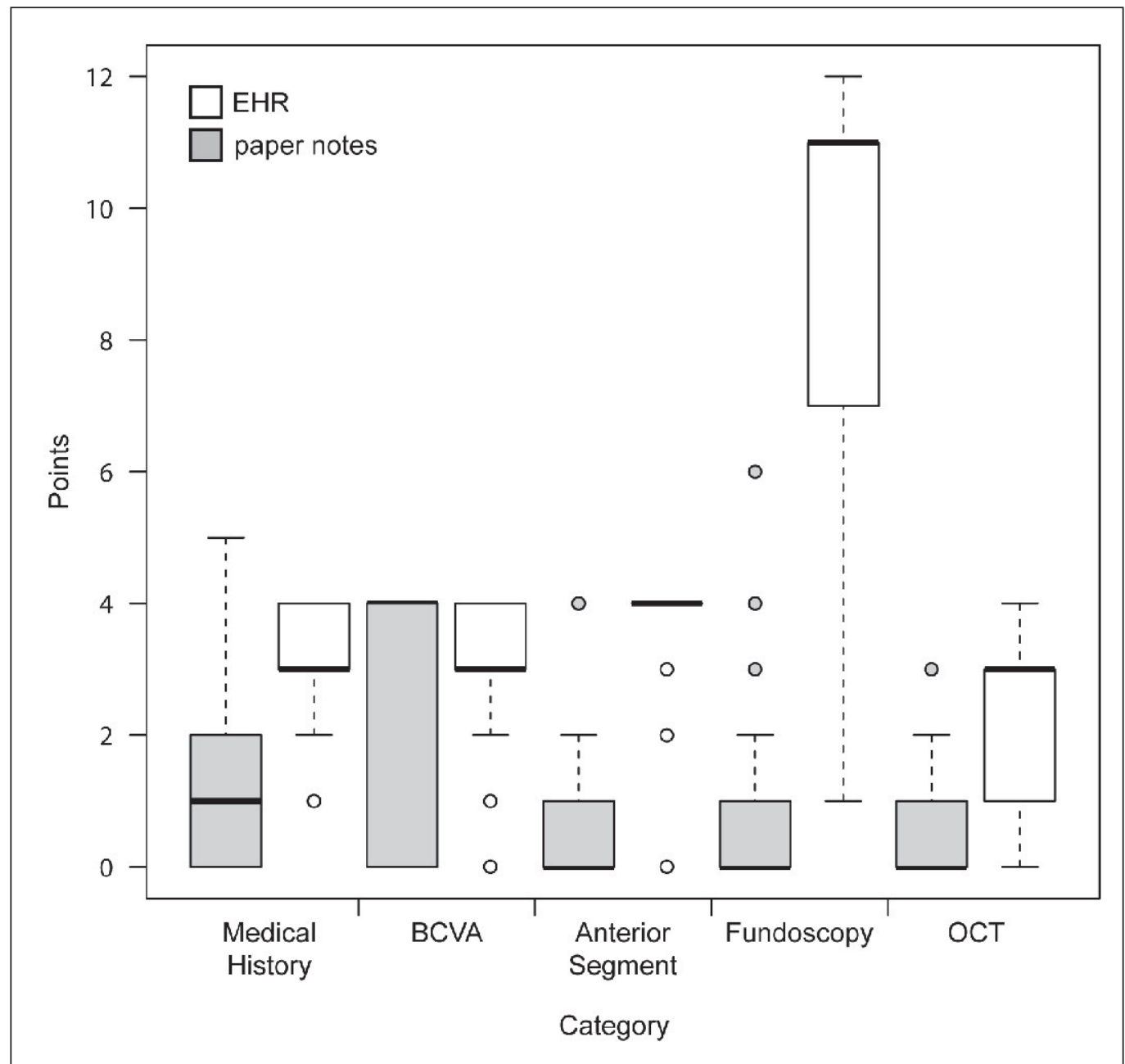

Fig. 2 Box-and-whisker plot of the mean completeness grouped by categories and displayed by documentation type. BCVA: Best-corrected visual acuity, OCT: Optical coherence tomography 


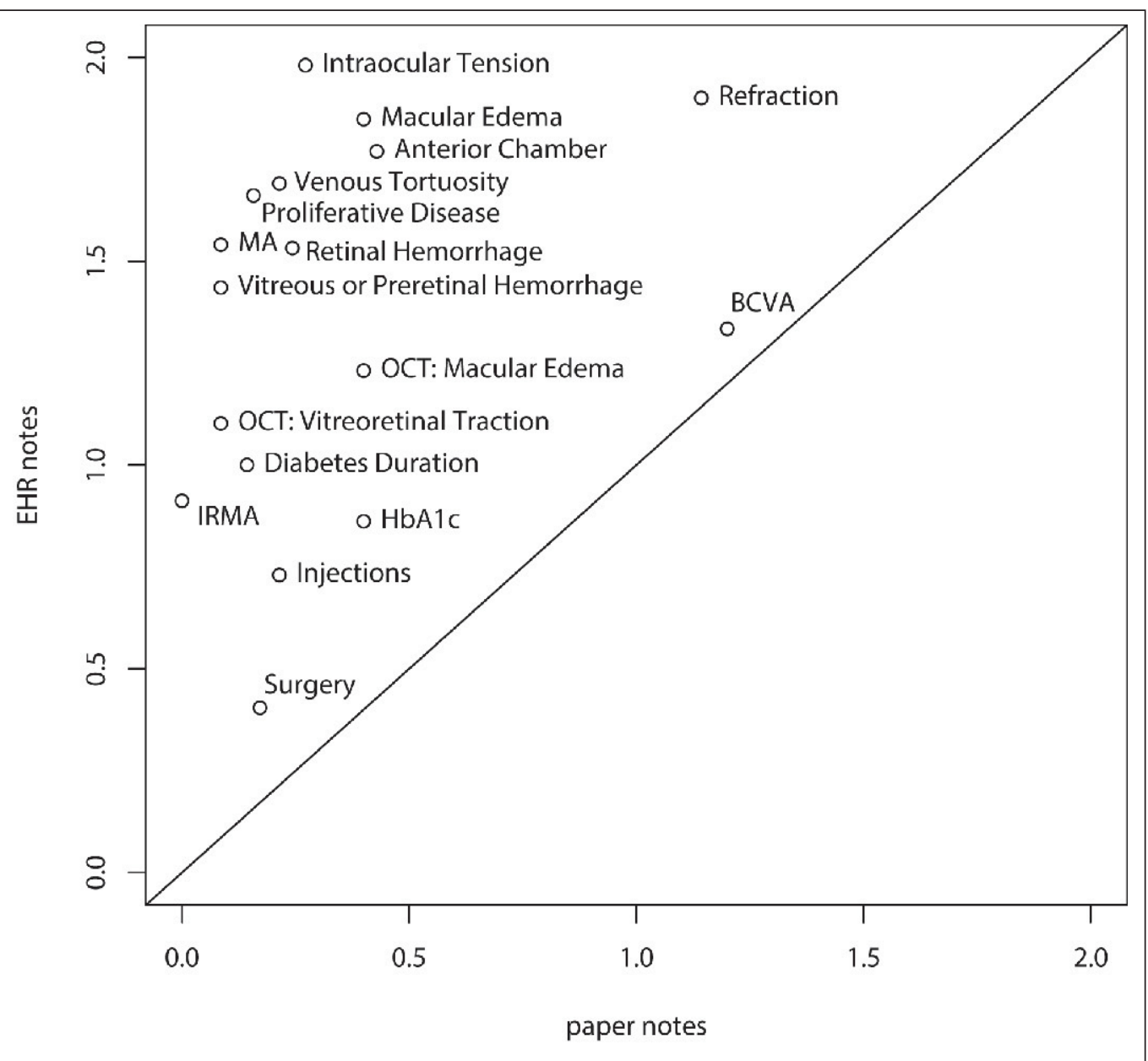

Fig. 3 Scatter plot of the mean scores on EHR and paper notes. BCVA: Best-corrected visual acuity, HbA1c: Hemoglobin A1c; percentage of glycosylated hemoglobin in blood, IRMA: intraretinal microvascular abnormalities, MA: microaneurysms, OCT: optical coherence tomography 
Table 1 Results

\begin{tabular}{|c|c|c|c|c|}
\hline \multirow[t]{2}{*}{ Category } & \multirow[t]{2}{*}{$\max }$. & \multicolumn{2}{|c|}{ mean score } & \multirow[t]{2}{*}{$p$} \\
\hline & & Paper & EHR & \\
\hline Medical History & 6 & 0.929 & 2.997 & $<0.001$ \\
\hline Diabetes duration & 1 & 0.143 & 1.000 & $<0.001$ \\
\hline $\mathrm{HbA1c}$ & 1 & 0.400 & 0.862 & $<0.001$ \\
\hline History of injections & 2 & 0.214 & 0.730 & $<0.001$ \\
\hline Ocular surgical history & 2 & 0.171 & 0.405 & $<0.001$ \\
\hline BCVA & 4 & 2.343 & 3.235 & $<0.001$ \\
\hline Refraction & 2 & 1.143 & 1.901 & $<0.001$ \\
\hline Visual acuity & 2 & 1.200 & 1.334 & $=0.247$ \\
\hline Anterior Segment and Tension & 4 & 0.700 & 3.75 & $<0.001$ \\
\hline Anterior chamber & 2 & 0.429 & 1.770 & $<0.001$ \\
\hline Intraocular tension & 2 & 0.271 & 1.981 & $<0.001$ \\
\hline Fundoscopy & 14 & 0.971 & 8.931 & $<0.001$ \\
\hline Macular edema & 2 & 0.400 & 1.849 & $<0.001$ \\
\hline Proliferative disease & 2 & 0.157 & 1.661 & $<0.001$ \\
\hline Retinal hemorrhage & 2 & 0.243 & 1.532 & $<0.001$ \\
\hline Venous tortuosity & 2 & 0.086 & 1.541 & $<0.001$ \\
\hline Microaneurysms & 2 & 0.214 & 1.691 & $<0.001$ \\
\hline IRMA & 2 & 0.000 & 0.912 & $<0.001$ \\
\hline Vitreous or preretinal hemorrhage & 2 & 0.086 & 1.436 & $<0.001$ \\
\hline OCT & 4 & 0.486 & 2.334 & $<0.001$ \\
\hline Macular edema & 2 & 0.400 & 1.232 & $<0.001$ \\
\hline Vitreoretinal traction & 2 & 0.086 & 1.102 & $<0.001$ \\
\hline SUM & 32 & 5.429 & 21.248 & $<0.001$ \\
\hline
\end{tabular}

BCVA: Best-corrected visual acuity, OCT: Optical coherence tomography, HbA1c: Hemoglobin A1c, percentage of glycosylated hemoglobin in blood, IRMA: Intra-retinal microvascular abnormalities 


\section{References}

1. Greenes RA. Medical Informatics. JAMA. American Medical Association 1990; 263(8):1114.

2. Richard S. Dick, Elaine B. Steen, and Don E. Detmer, Editors; Committee on Improving the Patient Record I of M. The Computer-Based Patient Record: An Essential Technology for Health Care, Revised Edition Natl Acad Press 1997

3. Furukawa MF, King J, Patel V, Hsiao C-J, Adler-Milstein J, Jha AK. Despite substantial progress In EHR adoption, health information exchange and patient engagement remain low in office settings. Health Aff (Millwood). 2014; (9):1672-1679.

4. Grabenbauer L, Skinner a., Windle J. Electronic Health Record Adoption - Maybe It's not about the Money. Appl Clin Inform 2011; 2(4): 4604-4671.

5. Hoerbst a., Ammenwerth E. Electronic health records: A systematic review on quality requirements. Methods Inf Med. $2010 ; 49(4): 320-336$.

6. Katzer R, Barton DJ, Adelman S, Clark S, Seaman EL, Hudson KB. Impact of implementing an EMR on physical exam documentation by ambulance personnel. Appl Clin Inform 2012; 3(3): 301-308.

7. Sanders DS, Lattin DJ, Read-Brown S, Tu DC, Wilson DJ, Hwang TS, Morrison JC, Yackel TR, Chiang MF. Electronic health record systems in ophthalmology: impact on clinical documentation. Ophthalmology 2013; 120(9): 1745-1755.

8. Pandit RR, Boland M V. The impact of an electronic health record transition on a glaucoma subspecialty practice. Ophthalmology 2013; 120(4): 753-760.

9. Sanders DS, Read-Brown S, Tu DC, Lambert WE, Choi D, Almario BM, Yackel TR, Brown AS, Chiang MF. Impact of an electronic health record operating room management system in ophthalmology on documentation time, surgical volume, and staffing. JAMA Ophthalmol 2014; 132(5): 586-592.

10. Dorda W, Wrba T, Duftschmid G, Sachs P, Gall W, Rehnelt C, Boldt G, Premauer W. ArchiMed: a medical information and retrieval system. Methods Inf Med 1999; 38(1): 16-24.

11. American Academy of Ophthalmology Retina/Vitreous Panel. Preferred Practice Pattern (R) Guidelines. Diabetic Retinopathy. San Francisco, CA: American Academy of Ophthalmology; 2014. Available from: www.aao.org/ppp 